DEMONSTRATIO MATHEMATICA

Vol. XLII No 42009

Belmesnaoui Aqzzouz, Khalid Bourass

\title{
SOME PROPERTIES OF POSITIVE WEAK DUNFORD-PETTIS OPERATORS ON BANACH LATTICES
}

\begin{abstract}
We characterize Banach lattices for which each positive weak DunfordPettis operator is weakly compact. As consequences, we obtain some interesting results on reflexive Banach lattices.
\end{abstract}

\section{Introduction and notation}

An operator $T$ from a Banach space $E$ into another $F$ is said to be weak Dunford-Pettis if the sequence $\left(y_{n}^{\prime}\left(T\left(x_{n}\right)\right)\right)$ converges to 0 whenever $\left(x_{n}\right)$ converges weakly to 0 in $E$ and $\left(y_{n}^{\prime}\right)$ converges weakly to 0 in $F^{\prime}$. The operator $T$ is called Dunford-Pettis if it carries weakly compact subsets of $E$ onto compact subsets of $F$. Note that each Dunford-Pettis operator is weak Dunford-Pettis but a weak Dunford-Pettis operator is not necessary Dunford-Pettis.

On the other hand, it is well known that a weakly compact operator is not necessary Dunford-Pettis. And as each Dunford-Pettis operator is weak Dunford-Pettis, it is natural to ask if each weakly compact operator is weak Dunford-Pettis. The answer is negative. In fact, since the Banach space $L^{2}([0,1])$ is reflexive, its identity operator is weakly compact, but it is not weak Dunford-Pettis.

Note that a Banach space $E$ has the Dunford-Pettis property if every weakly compact operator defined on $E$ (and taking their values in another Banach space) is Dunford-Pettis and hence weak Dunford-Pettis.

Conversely, a weak Dunford-Pettis operator is not always weakly compact. In fact, since the Banach space $l^{1}$ has the Dunford-Pettis property, its identity operators is weak Dunford-Pettis but it is not weakly compact.

2000 Mathematics Subject Classification: 46A40, 46B40, 46B42.

Key words and phrases: weak Dunford-Pettis operator, weakly compact operator, order continuous norm, KB-space, reflexive space. 
Our goal in this paper is to characterize Banach lattices for which each positive weak Dunford-Pettis operator is weakly compact. More precisely, we will prove that if $E$ is a Dedekind $\sigma$-complete Banach lattice, then each positive weak Dunford-Pettis operator from $E$ into $E$ is weakly compact if and only if $E$ is reflexive (Theorem 2.1). Next, whenever $E$ and $F$ are two Dedekind $\sigma$-complete Banach lattices, we will show that if each positive weak Dunford-Pettis operator from $E$ into $F$ is weakly compact, then $E$ is reflexive or $F$ has an order continuous norm (Theorem 2.2). After that, by an example, we will establish that the second necessary condition of Theorem 2.2 is not sufficient (Remark 2.4). Finally, we will give some consequences (Corollaries 2.3 and 2.5).

To state our results, we need to fix some notation and recall some definitions. A vector lattice $E$ is an ordered vector space in which $\sup (x, y)$ exists for every $x, y \in E$. A vector lattice is said to be Dedekind $\sigma$-complete if every nonempty countable subset that is bounded from above has a supremum. A Banach lattice is a Banach space $(E,\|\|$.$) such that E$ is a vector lattice and its norm satisfies the following property: for each $x, y \in E$ such that $|x| \leq|y|$, we have $\|x\| \leq\|y\|$. If $E$ is a Banach lattice, its topological dual $E^{\prime}$, endowed with the dual norm and the dual order, is also a Banach lattice. A norm $\|$.$\| of a Banach lattice E$ is order continuous if for each generalized sequence $\left(x_{\alpha}\right)$ such that $x_{\alpha} \downarrow 0$ in $E$, the sequence $\left(x_{\alpha}\right)$ converges to 0 for the norm $\|$.$\| where the notation x_{\alpha} \downarrow 0$ means that the sequence $\left(x_{\alpha}\right)$ is decreasing, its infimum exists and $\inf \left(x_{\alpha}\right)=0$. A Banach lattice $E$ is said to be an AM-space if for each $x, y \in E$ such that $\inf (x, y)=0$, we have $\|x+y\|=\max \{\|x\|,\|y\|\}$.

We will use the term operator $T: E \longrightarrow F$ between two Banach lattices to mean a bounded linear mapping. It is positive if $T(x) \geq 0$ in $F$ whenever $x \geq 0$ in $E$. It is well known that each positive linear mapping on a Banach lattice is continuous.

For unexplained terminology on Banach lattice theory and positive operators, we refer the reader to [1].

\section{Main results}

A Banach lattice $E$ is said to be a KB-space, whenever every increasing norm bounded sequence of $E^{+}$is norm convergent. As an example, each reflexive Banach lattice is a $\mathrm{KB}$-space.

Note that each KB-space has an order continuous norm, but a Banach lattice with an order continuous norm is not necessary a KB-space. In fact, the Banach lattice $c_{0}$ has an order continuous norm but it is not a KB-space. However, if $E$ is a Banach lattice, the topological dual $E^{\prime}$ is a KB-space if and only if its norm is order continuous. 
Let us recall from Theorem 5.16 of Schaefer [3], that a Banach lattice $E$ is reflexive if and only if the norms of its topological dual $E^{\prime}$ and of its topological bidual $E^{\prime \prime}$ are order continuous.

Our principal characterization is the following.

Theorem 2.1. Let $E$ be a Dedekind $\sigma$-complete Banach lattice. Then the following assertions are equivalent:

1) $E$ is reflexive.

2) Each positive weak Dunford-Pettis operator from $E$ into $E$ is compact.

3) For all operators $S, T: E \longrightarrow E$ such that $0 \leq S \leq T$ and $T$ is weak Dunford-Pettis, $S$ is compact.

4) Each positive weak Dunford-Pettis operator from $E$ into $E$ is weakly compact.

5) For each positive weak Dunford-Pettis operator $T: E \longrightarrow E$, the operator product $T^{2}$ is weakly compact.

Proof. 1) $\Longrightarrow 2$ ) Let $T: E \longrightarrow E$ be a positive weak Dunford-Pettis operator. Since $E$ is reflexive, its identity operator $I d_{E}: E \longrightarrow E$ is weakly compact. Then it follows from Theorem 5.99 of [1] that the composed operator $T=I d_{E} \circ T: E \longrightarrow E$ is Dunford-Pettis. Finally, Theorem 5.81 of [1] implies that $T$ is compact.

2) $\Longrightarrow 3$ ) Let $S, T: E \longrightarrow E$ be two operators such that $0 \leq S \leq T$ and $T$ is weak Dunford-Pettis. By Theorem 4.5 of Kalton-Saab [2], $S$ is weak Dunford-Pettis and hence it is compact.

$3) \Longrightarrow 4$ ) Let $T: E \longrightarrow E$ be a positive weak Dunford-Pettis operator. Since $0 \leq T \leq T$, it follows that $T$ is compact, and hence it is weakly compact.

4) $\Longrightarrow 5$ ) Obvious.

$5) \Longrightarrow 1$ ) Step 1. We prove that the norm of $E$ is order continuous. If not, it follows from the proof of Theorem 1 of Wickstead [4] that $E$ contains a closed sublattice isomorphic to $l^{\infty}$ and there is a positive projection $P$ : $E \rightarrow l^{\infty}$. Let $i: l^{\infty} \rightarrow E$ be the canonical injection of $l^{\infty}$ into $E$. Since $l^{\infty}$ has the Dunford-Pettis property, the operator $T$ defined by

$$
T=i \circ P: E \rightarrow l^{\infty} \rightarrow E
$$

is positive and weak Dunford-Pettis. But, its second power

$$
(T)^{2}: E \longrightarrow l^{\infty} \longrightarrow E \longrightarrow l^{\infty} \longrightarrow E,
$$

which coincides with $T$, is not weakly compact. If not, the operator

$$
P \circ T \circ i: l^{\infty} \rightarrow E \rightarrow l^{\infty} \rightarrow E \rightarrow l^{\infty}
$$


would be weakly compact. But $P \circ T \circ i$, which is just the identity operator $I d_{l^{\infty}}$, is not weakly compact. This presents a contradiction and hence $E$ has an order continuous norm.

Step 2. We prove that $E$ is a KB-space. If not, as the norm of $E$ is order continuous, it follows from the proof of Theorem 2 of Wnuk [5] the existence of a closed sublattice of $E$, which is isomorphic to $c_{0}$ and a positive projection $P_{1}: E \longrightarrow c_{0}$. Let $i_{1}: c_{0} \longrightarrow E$ be the canonical injection of $c_{0}$ into $E$. Since $c_{0}$ has the Dunford-Pettis property, the operator defined by

$$
T_{1}=i_{1} \circ P_{1}: E \longrightarrow c_{0} \longrightarrow E,
$$

is weak Dunford-Pettis. But, its second power

$$
\left(T_{1}\right)^{2}: E \longrightarrow c_{0} \longrightarrow E \longrightarrow c_{0} \longrightarrow E,
$$

which coincides with $T_{1}$, is not weakly compact (if not, the operator $P_{1}$ 。 $T_{1} \circ i_{1}=I d_{c_{0}}: c_{0} \longrightarrow c_{0}$ would be weakly compact and this is false). This presents a contradiction and hence $E$ is a KB-space.

Step 3. We show that the norm of the topological dual $E^{\prime}$ is order continuous. If not, it follows from the proof of Theorem 1 of Wickstead [4] the existence of a sublattice $H$ of $E$ isomorphic to $l^{1}$ and a positive projection $P_{2}: E \longrightarrow l^{1}$. Let $T_{2}$ be the operator defined by $T_{2}=i_{2} \circ P_{2}: E \rightarrow l^{1} \rightarrow E$ where $i_{2}: l^{1} \longrightarrow E$ is the canonical injection of $l^{1}$ into $E$. Since $l^{1}$ has the Dunford-Pettis property, $T_{2}$ is weak Dunford-Pettis but $\left(T_{2}\right)^{2}=T_{2}$ is not weakly compact. If not, the operator $P_{2} \circ T_{2} \circ i_{2}=I d_{l^{1}}$ would be weakly compact, and this gives a contradiction.

Whenever $E \neq F$ in Theorem 2.1, we obtain the following necessary conditions.

Theorem 2.2. Let $E$ and $F$ be two Dedekind $\sigma$-complete Banach lattices. If each positive weak Dunford-Pettis operator from $E$ into $F$ is weakly compact, then one of the following assertions is valid:

1) $E$ is reflexive.

2) $F$ has an order continuous norm.

Proof. It suffices to prove that if the norm of $F$ is not order continuous, then $E$ is reflexive i.e. $E$ and $E^{\prime}$ are KB-spaces (Theorem 5.16 of Schaefer [3]). Assume that the norm of $F$ is not order continuous and hence it is not reflexive.

Step 1. We prove that $E^{\prime}$ has an order continuous norm i.e. $E^{\prime}$ is a KB-space. If not, it follows from the proof of Theorem 1 of Wickstead [4] the existence of a sublattice $H$ of $E$ which is isomorphic to $l^{1}$ and a positive projection $P: E \rightarrow l^{1}$. Also, since $F$ is not reflexive, its closed unit ball $B_{F}$ is not weakly compact and then there exists a sequence $\left(y_{n}\right)$ in $\left(B_{F}\right)^{+}$ 
which does not have any weakly convergent subsequence (Eberlein-Smulian's Theorem).

Consider the operator $S$ defined by

$$
S: l^{1} \rightarrow F,\left(\lambda_{n}\right) \mapsto \sum_{n=1}^{\infty} \lambda_{n} y_{n} .
$$

Since the Banach space $l^{1}$ has the Dunford-Pettis property, the positive operator $S \circ P$ is weak Dunford-Pettis. But it is not weakly compact, indeed, as the sequence $\left(S\left(e_{n}\right)\right)=\left(y_{n}\right)$ does not have any weakly convergent subsequence, where $\left(e_{n}\right)$ is the canonical basis of $l^{1}$. This presents a contradiction, and hence $E^{\prime}$ is a KB-space.

Step 2. We prove that $E$ is reflexive. For this it suffices to show that $E$ is a $\mathrm{KB}$-space.

In the first time, we prove that the norm of $E$ is order continuous. If not, it follows from the proof of Theorem 1 of Wickstead [4] that $E$ (resp. $F$ ) contains a closed sublattice isomorphic to $l^{\infty}$ and there is a positive projection $P_{1}: E \rightarrow l^{\infty}$ (resp. $Q_{1}: F \rightarrow l^{\infty}$ ).

Let $i_{1}: l^{\infty} \rightarrow E$ (resp. $j_{1}: l^{\infty} \rightarrow F$ ) be the canonical injection of $l^{\infty}$ into $E$ (resp. into $F$ ). Since $l^{\infty}$ has the Dunford-Pettis property, the operator $T_{1}$ defined by

$$
T_{1}=j_{1} \circ P_{1}: E \rightarrow l^{\infty} \rightarrow F
$$

is positive and weak Dunford-Pettis, but it is not weakly compact. If not, the operator

$$
Q_{1} \circ T_{1} \circ i_{1}: l^{\infty} \rightarrow E \rightarrow l^{\infty} \rightarrow F \rightarrow l^{\infty}
$$

would be weakly compact. But $Q_{1} \circ T_{1} \circ i_{1}=I d_{l \circ}$ is not weakly compact. This presents a contradiction and hence $E$ has an order continuous norm.

In the second time, we establish that $E$ is a KB-space. If not, as the norm of $E$ is order continuous, it follows from the proof of Theorem 2 of Wnuk [5] the existence of a closed sublattice of $E$ isomorphic to $c_{0}$ and a positive projection $P_{2}: E \rightarrow c_{0}$. Let $i_{2}: c_{0} \rightarrow E$ be the canonical injection of $c_{0}$ into $E$.

Also, as the norm of $F$ is not order continuous, then $F$ contains a closed sublattice isomorphic to $c_{0}$. Let $j_{2}: c_{0} \rightarrow F$ be the canonical injection. If $T_{2}$ is the operator defined by

$$
T_{2}=j_{2} \circ P_{2}: E \rightarrow c_{0} \rightarrow F .
$$

This operator is weak Dunford-Pettis, but it is not weakly compact. If not, the operator

$$
T_{2} \circ i_{2}=j_{2}: c_{0} \rightarrow F
$$


would be weakly compact, but this is not true. This presents a contradiction and hence $E$ is a KB-space. This ends the proof of the Theorem.

As a consequence of Theorem 5.24 of [1] and Theorem 2.2, we obtain the following characterization of reflexive Banach lattices:

Corollary 2.3. Let $E$ and $F$ be two Dedekind $\sigma$-complete Banach lattices such that $F$ is an infinite-dimensional AM-space with unit. Then the following assertions are equivalent:

1) $E$ is reflexive.

2) Each operator from $E$ into $F$ is weakly compact.

3) Each positive weak Dunford-Pettis operator from $E$ into $F$ is weakly compact.

Proof. 1) $\Longrightarrow$ 2) Follows from Theorem 5.24 of [1].

2) $\Longrightarrow 3$ ) Obvious.

$3) \Longrightarrow 1$ ) The norm of the Banach lattice $F$ is not order continuous. Indeed, if the norm of the Banach lattice $F$ is order continuous, as $F$ is an AM-space with unit, it follows from Theorem 5.10 of Schaefer [3] that the closed unit ball $B_{F}=[-e, e]$ is weakly compact and hence $E$ is reflexive. Now, by Corollary 2 of ([3], paragraph (9.9)), the Banach lattice $F$ is finitedimensional. This gives a contradiction. Finally, since the norm of the Banach lattice $F$ is not order continuous it follows from Theorem 2.2 that $E$ is reflexive.

REMARK 2.4. The second necessary condition of Theorem 2.2 is not sufficient. In fact, if we take $E=F=c_{0}$, it is well known that $c_{0}$ is not reflexive. But since $c_{0}$ has the Dunford-Pettis property, its identity operator $I d_{c_{0}}: c_{0}$ $\longrightarrow c_{0}$ is weak Dunford-Pettis but it is not weakly compact. Although, the norm of $c_{0}$ is order continuous.

However, we have the following property:

Corollary 2.5. Let $E$ be an infinite-dimensional AM-space with unit and $F$ a Banach lattice. Then the following assertions are equivalent:

1) Each positive operator from $E$ into $F$ is weakly compact.

2) Each positive weak Dunford-Pettis operator from $E$ into $F$ is weakly compact.

3) The norm of $F$ is order continuous.

Proof. 1) $\Longrightarrow 2$ ) Obvious.

$2) \Longrightarrow 3$ ) By Corollary 2 of ([3], paragraph (9.9)), the AM-space $E$ is not reflexive, and hence it follows from Theorem 2.2, that the norm of $F$ is order continuous. 
$3) \Longrightarrow 1$ ) Let $T$ be a positive operator from $E$ into $F$. Since $E$ is an $\mathrm{AM}$-space with unit $e$, we have $B_{E}=[-e, e]$ and then $T\left(B_{E}\right)=T([-e, e]) \subset$ $[-T(e), T(e)]$. As the norm of $F$ is order continuous, Theorem 5.10 of [3] implies that $[-T(e), T(e)]$ is weakly compact. And hence $T$ is weakly compact.

REMARK 2.6. If the Banach lattice $E$ is an AM-space without unit, the precedent Corollary is false. In fact, if we take $E=F=c_{0}$, the identity operator $I d_{c_{0}}: c_{0} \longrightarrow c_{0}$ is weak Dunford-Pettis but it is not weakly compact.

\section{References}

[1] C. D. Aliprantis, O. Burkinshaw, Positive operators, Reprint of the 1985 original. Springer, Dordrecht, 2006.

[2] N. J. Kalton, P. Saab, Ideal properties of regular operators between Banach lattices, Illinois J. Math. Vol. 29, 3 (1985), 382-400.

[3] H. H. Schaefer, Banach Lattices and Positive Operators, Springer-Verlag, Berlin and New York, 1974.

[4] A. W. Wickstead, Converses for the Dodds-Fremlin and Kalton-Saab theorems, Math. Proc. Cambridge Philos. Soc. 120 (1996), 175-179.

[5] W. Wnuk, Some characterizations of Banach lattices with the Schur property, Congress on Functional Analysis (Madrid, 1988). Rev. Mat. Univ. Complut. Madrid 2 (1989), suppl., $217-224$.

Belmesnaoui Aqzzouz

UNIVERSITÉ MOHAMMED V-SOUISSI

FACULTÉ DES SCIENCES ECONOMIQUES

JURIDIQUES ET SOCIALES

DÉPARTEMENT D'ECONOMIE

B.P. 5295, SALAELJADIDA

MOROCCO

E-mail: baqzzouz@hotmail.com

Khalid Bourass

UNIVERSITÉ IBN TOFAIL

FACULTÉ DES SCIENCES

DÉPARTEMENT DE MATHÉMATIQUES

B.P. 133, KÉNITRA, MOROCCO

Received March 9, 2008; revised version March 14, 2009. 
